\title{
Possible implementation of adiabatic quantum algorithm with superconducting flux qubits
}

\author{
M. Grajcar, ${ }^{1,2, *}$ A. Izmalkov, ${ }^{2,3}$ and E. Il'ichev ${ }^{2}$ \\ ${ }^{1}$ Department of Solid State Physics, Comenius University, SK-84248 Bratislava, Slovakia \\ ${ }^{2}$ Institute for Physical High Technology, P.O. Box 100239, D-07702 Jena, Germany \\ ${ }^{3}$ Moscow Engineering Physics Institute (State University), Kashirskoe sh. 31, 115409 Moscow, Russia
}

(Dated: November 15, 2018)

\begin{abstract}
We show that an $L C$ parametric transducer can be effectively used to monitor an adiabatic evolution of the superconducting flux qubit. We propose a new scheme to measure the qubit's state, which is a quantum nondemolition measurement. The scheme can be easily extended to a threequbit system, and allows the reading out of the qubits' states while the system remains in the ground state. An implementation of the adiabatic quantum algorithm MAXCUT for three superconducting flux qubits is discussed.
\end{abstract}

PACS numbers: 85.25.Cp, 85.25.Dq, 03.67.Lx

\section{INTRODUCTION}

Ten years ago Peter Shor demonstrated theoretically ${ }^{1}$ that a quantum computer can factor large numbers much more effectively than a classical one. This discovery started an enormous effort to find a physical system which would be a suitable qubit, the building block of a quantum computer. Qubits are effectively two-level systems with controlled parameters. There are many systems in physics which can play the role of a qubit. One of them is a superconducting flux qubit which can be realized as a superconducting loop with low inductance $L_{q}$ interrupted by three Josephson junctions. Its properties have already been analysed ${ }^{2,3}$ and experimentally verified. ${ }^{4}$ Superconducting qubits have several advantages over qubits based on microscopic systems: they are scalable and can be accessed more easily and controlled individually. Moreover, aluminum technology, widely exploited for the preparation of conventional silicon devices, can be used.

Recently, several groups succeeded in demonstrating coherent macroscopic tunneling and Rabi oscillations in superconducting qubits. This can be considered as the first important step towards quantum computer realization. ${ }^{5-7}$ Most of them were time domain measurements, which are supposed to be important for quantum computing, since the much effort has been made in the direction of building a quantum computer based on a universal set of gates. However, in order to run an algorithm on such a universal quantum computer, quantum error corrections should be implemented. For a solid state qubit the error rate is only slightly below the threshold required for fault-tolerant computation. This places tremendous requirements on the hardware: ${ }^{8}$ the number of physical qubits should be larger than $10^{4}$ and teleportation between each two qubits should be possible. On the other hand, the new scheme of quantum computation based on adiabatic quantum evolution, which has been proposed by Farhi et al. ${ }^{9}$ could solve tasks beyond the reach of present-day classical computers for a very moder- ate number of qubits ( $\gtrsim 30)$. Very recently a scalable superconducting architecture for adiabatic quantum computation was proposed which requires nearest-neighbor coupling only. ${ }^{10}$ Moreover, Aharonov et al. ${ }^{11}$ have shown that adiabatic quantum computation is equivalent to standard quantum computation. From an experimental point of view the adiabatic quantum algorithm MAXCUT was demonstrated by an NMR technique on three qubit systems. ${ }^{12}$ In this paper, we propose a specific implementation for adiabatic quantum computing with a set of coupled superconducting flux qubits, which is possible to realize with the present state of the art. We show that a parametric transducer can be effectively used to read out the results of the adiabatic evolution algorithm.

\section{PARAMETRIC TRANSDUCER AS A QND READOUT FOR ADIABATIC QUANTUM COMPUTATION}

Parametric transducers have been shown to be very sensitive instruments, that can overcome the standard quantum limit. ${ }^{13}$ The precision of the measurement of small changes of the dielectric susceptibility by a capacity transducer is of the order of $10^{-10}$. In addition, a parametric transducer can work in a regime that satisfies the criteria of quantum nondemolition (QND) measurements. Usually, an electromagnetic auto-oscillator is used as a key element of a parametric transducer since the frequency can be measured with a very high accuracy. The scheme of a parametric transducer is shown in Fig.1, it contain a high quality $L C$ resonator connected to an amplifier. ${ }^{14}$ The resonant frequency of the $L C$ circuit depends on both the inductance $L$ and the capacitance $C$ by the relation $\omega_{r}=1 / \sqrt{L C}$. In our experiments, typically $\omega_{r} / 2 \pi \sim 30 \mathrm{MHz}$. This satisfies $\omega_{r} \ll \omega_{q}$, where $\omega_{q}$ is the transition frequency between the ground and first excited energy level of the qubit. Thus, the magnetic susceptibility of the qubit placed in a resonator can be measured from the shift of the resonance frequency. It can be easily shown ${ }^{13}$ that the tangent of the phase shift 


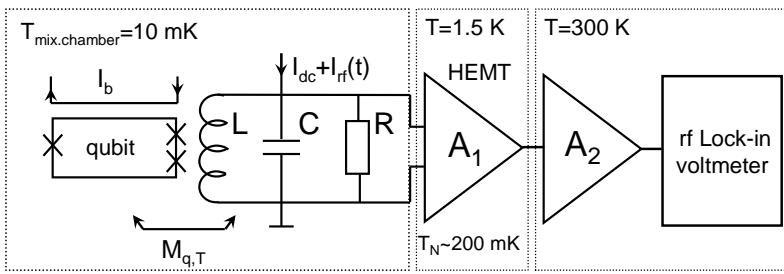

FIG. 1: Scheme of a parametric transducer inductively coupled to a superconducting flux qubit. The $r f$ voltage across the tank is amplified by a cooled HEMT amplifier thermally linked to a $1 \mathrm{~K}$ pot. ${ }^{14}$ After room temperature amplification the signal is detected by an $r f$ lock-in voltmeter. Both the amplitude and phase of the $r f$ voltage are measured as a function of the external magnetic flux applied to the qubit produced by the currents $I_{d c}$ and $I_{b}$ through a coil and a wire, respectively.

$\theta$ between the voltage across the tank and driving current is proportional to the real part of $a c$ susceptibility $\chi^{\prime}$

$$
\tan \theta=-k^{2} Q \chi^{\prime}
$$

where $0<k<1$ is the coupling coefficient between the resonator and sample. The ideas behind a parametric transducer were also used in the design of an $r f$-SQUID by Silver and Zimmermann. ${ }^{15}$ It was shown theoretically that an $r f$-SQUID can achieve the quantum limit. ${ }^{16}$ Therefore, the parametric transducer is a suitable readout device for superconducting flux qubits.

The magnetic susceptibility of the superconducting flux qubit is ${ }^{17}$

$$
\chi^{\prime}=L_{q} I_{q}^{2} \frac{\Delta^{2}}{\left(\Delta^{2}+\varepsilon^{2}\right)^{3 / 2}} \tanh \left(\frac{\sqrt{\Delta^{2}+\varepsilon^{2}}}{T}\right)
$$

where $\Delta$ is the tunneling amplitude, $L_{q}$ is the inductance of the flux qubit, $I_{q}$ is the persistent current in the qubit, $T$ is the temperature, and $\varepsilon=\Phi_{0} I_{q} f$ is the bias of the qubit, where $f$ is the deviation from degeneracy defined in terms of internal magnetic flux in the qubit as $f=\Phi_{i} / \Phi_{0}-0.5$. By using Eqs. (1),(2) the persistent current and the tunneling amplitude can be determined experimentally by measuring the resonator phase as a function of the external magnetic flux $\Phi_{e} \cdot{ }^{18}$ The function $\chi^{\prime}(f)$ (Eq. 2) has a simple form, and it is easily seen that $\chi^{\prime}(f)$ exhibits a peak at the degeneracy point $f=0$. If the temperature $T \ll \Delta$, the explicit equations for the persistent current and the tunneling amplitude can be readily derived

$$
\begin{gathered}
I_{q}=\frac{\Phi_{0}}{L_{q}} \frac{\chi_{a}^{\prime} f_{F W H M}}{2 \sqrt{2^{2 / 3}-1}} \\
\Delta=\Phi_{0} I_{q} \frac{f_{F W H M}}{2 \sqrt{2^{2 / 3}-1}}
\end{gathered}
$$

where $\chi_{a}^{\prime}$ and $f_{\mathrm{FWHM}}$ are the peak amplitude and the full width at half maximum (FWHM), respectively.

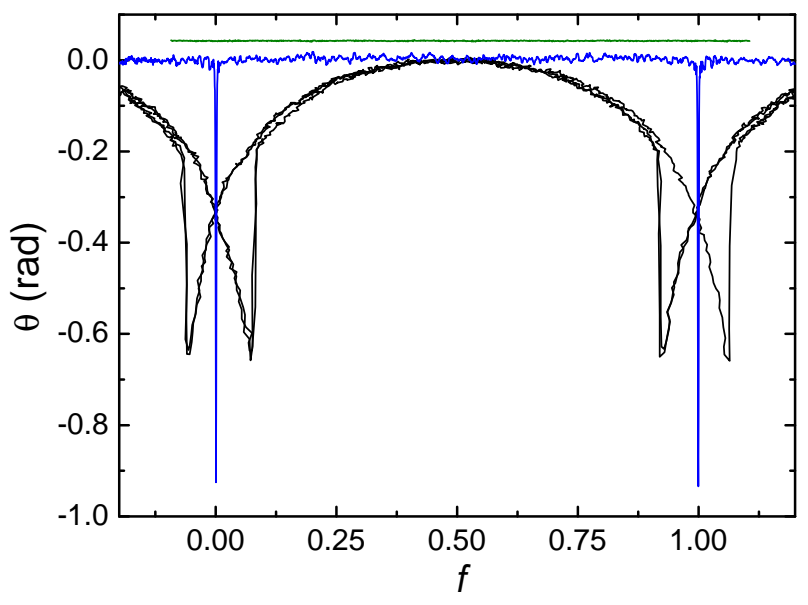

FIG. 2: The phase shift $\theta$ between the bias current $I_{r f}$ and the $r f$ voltage of the parametric transducer inductively coupled to the superconducting flux qubit as a function of the internal magnetic flux in the qubit. The curve with hysteretic behavior (black curve) corresponds to the 'qubits' with a large ratio $g=$ $E_{J} / E_{C} \sim 10^{3}$ (classical regime). The straight line (vertically shifted for clarity) and the non-hysteretic line correspond to qubits with $g \approx 60$, and $\alpha=0.9$ and $\alpha=0.8$, respectively.

Here we would like to point out that the measurement by means of a parametric transducer is a quantum nondemolition measurement, because the qubit is staying in its ground state the entire time of the measurement, as the resonant frequency of the resonator $\omega_{r}$ is much lower than the transition frequency $\omega_{q}$. The output signal of the parametric transducer contains information about the amplitude of the persistent current, but holds no information about the phase of the rapidly oscillating persistent current. A parametric transducer cannot even distinguish whether the current flows clockwise or counterclockwise. This can be directly seen for 'qubits' in the classical regime (see the hysteretic curve in Fig. 2). Exactly at the degeneracy point $f=0$, the two branches of the hysteretic curves corresponding to current flowing clockwise and counterclockwise cross, i.e. the transducer gives the same signal. The reason for this is that the operator probed by the inductive transducer is $\sigma_{x}$ as we show below. In this sense, such a readout is complemental to the SQUID readout which measures $\sigma_{z}$ ( $\sigma_{x}$ and $\sigma_{z}$ are Pauli matrices) to make the analogy with Stern-Gerlach apparatus complete. ${ }^{19}$ More formally, the Hamiltonian of a qubit-resonator system at the degeneracy point $f=0$ can be written in the form ${ }^{20}$

$$
H=H_{r}+H_{q}+H_{i n t}=\hbar \omega_{r} b^{\dagger} b+\Delta \sigma_{x}+\gamma\left(b^{\dagger}+b\right) \sigma_{z}
$$

where $b^{\dagger}, b$ are creation and annihilation operators, respectively, of the photon field in the resonator, $\gamma=$ $k \sqrt{\hbar \omega_{r} L_{q}} I_{q}$ is the coupling energy between the resonator and qubit, and $\sigma_{x}$ and $\sigma_{z}$ are Pauli matrices of the natural basis of the qubit (i.e. the two eigenstates of operator $\sigma_{z}$ correspond to the currents flowing clockwise and 
counterclockwise). After unitary transformation

$$
U_{1}=\frac{1}{\sqrt{2}}\left(\begin{array}{rr}
1 & 1 \\
1 & -1
\end{array}\right),
$$

the Hamiltonian (5) takes the form

$$
U_{1} H U_{1}^{\dagger}=\hbar \omega_{r} b^{\dagger} b+\Delta \sigma_{z}^{\prime}+\gamma\left(b^{\dagger} \sigma_{-}^{\prime}+b \sigma_{+}^{\prime}\right)
$$

where

$$
\sigma_{+}^{\prime}=\left(\begin{array}{ll}
0 & 1 \\
0 & 0
\end{array}\right), \sigma_{-}^{\prime}=\left(\begin{array}{ll}
0 & 0 \\
1 & 0
\end{array}\right)
$$

are spin-flip operators and $\sigma_{z}^{\prime}$ is the Pauli matrix in the eigenbasis of the qubit at the degeneracy point. Following the approach in Ref. 21, after a second unitary transformation

$$
U_{2}=\exp \left(\frac{\gamma}{2 \Delta}\left(b \sigma_{+}^{\prime}-b^{\dagger} \sigma_{-}^{\prime}\right)\right),
$$

and by expanding to second order in $\gamma / \Delta$, the transformed Hamiltonian $H^{\prime}=U_{2} U_{1} H U_{1}^{\dagger} U_{2}^{\dagger}$ is

$$
\frac{H^{\prime}}{\hbar \omega_{r}}=\left(1-k^{2} \frac{W_{q}}{\Delta} \sigma_{z}^{\prime}\right) b^{\dagger} b+\left(\frac{\Delta}{\hbar \omega_{r}}-\frac{k^{2}}{2} \frac{W_{q}}{\Delta}\right) \sigma_{z}^{\prime}
$$

where $W_{q}=L_{q} I_{q}^{2} / 2$ is the magnetic energy of the qubit. Experimentally, a shift of the resonant frequency of the resonator, which depends on the qubit state, is measured. This shift is determined by the first term of Eq. 10, i.e. the measured observable is $\sigma_{z}^{\prime}$, and one can readily find that the sufficient condition for QND measurement $\left[\sigma_{z}^{\prime}, H^{\prime}\right]=0$ is satisfied. ${ }^{13}$ Provided that the coupling between resonator and qubit is small $\sigma_{z}^{\prime}$ corresponds to $\sigma_{x}$ in the original basis. This means that the resonator measures the observable $\sigma_{x}$ at the degeneracy point in contrast with the SQUID which measures $\sigma_{z}$. Let us point out additional difference between resonator and SQUID measurement. The SQUID measurement makes a projection of the spin into the $z$-axis, i.e. at the degeneracy point the qubit is localized in one of the classical states after the measurement, and the SQUID gives a signal corresponding to this state. This measurement is nonQND since the SQUID is coupled directly to the oscillating variable. ${ }^{22}$ On the other hand, the resonator gives no signal if the qubit is in the eigenstate of the operator $\sigma_{z}$, i.e. the resonator does not perform a measurement, and therefore, does not disturb the qubit. Such a readout method has a clear advantage in the case of adiabatic quantum computing. The qubit remains in its ground state also after the measurement, i.e. the measurement of one qubit does not spoil the result of the adiabatic evolution. However, it should be noted that this statement is valid only if the amplitude of the circulating current in the resonator is small enough to avoid Landau-Zener transitions. Nevertheless, as we have shown theoretically in Ref. 23 and experimentally demonstrated in section III B, the noise temperature of the cooled amplifier ${ }^{14}$ enables one to fulfill this condition.
The readout procedure could be as follows; let us suppose that the qubit is in the state $|1\rangle$ (i.e. $f>0$, see Fig. 3). If the internal magnetic flux in the qubit is changing towards zero, then the qubit is moving through its degeneracy point $(f=0)$ where two classical energy levels cross (dashed lines in Fig. 3). At this point the qubit is in the superposition of the states $|0\rangle$ and $|1\rangle$ where the magnetic susceptibility of the qubit changes rapidly. Thus, the inductive transducer gives a considerable signal. On the other hand, if the qubit is in the state $|0\rangle$ (i.e. $f<0$ ), one should increase the external magnetic flux to move the qubit to the degeneracy point. If we do not know the state of the qubit at $f=f_{0}$ we can sweep the external magnetic flux in order to change the internal magnetic flux in the qubit around this point and from the response of the parametric transducer we can determine whether the qubit was in state $|0\rangle$ or $|1\rangle$ (signal is observed for $f>f_{0}$ or $f<f_{0}$, respectively). In the next section we will show numerically that the qubits can be readout one after another while staying all the time in the ground state of the system.

\section{ADIABATIC EVOLUTION}

\section{A. Theory}

The idea of quantum computation by adiabatic evolution is very simple but, surprisingly, was discovered only recently. ${ }^{9,10}$ It is based on the fact that, in practice, it is very difficult to find a ground state of certain Hamiltonians. Such a task belongs to the set of non-polynomial time (NP) problems. On the other hand, some Hamiltonians have a trivial ground state which is easy to find. Let us assume that the Hamiltonian of $N$ qubits $H(p)$ can be externally controlled by the parameter $p$ and that its ground state is separated from the first excited state by the energy gap $g(p)=E_{1}(p)-E_{0}(p)\left(E_{0}, E_{1}\right.$ are the two lowest eigenvalues of the Hamiltonian $H(p))$. Provided that the ground state of the initial Hamiltonian $H_{I}=H(p=0)$ can be easily found, we can construct it and then change the parameter $p$ slowly from $p=0$ to $p=1$. If we do it sufficiently slowly, i.e. in a time $\tau \gg$ $\hbar \varepsilon_{\max } / g_{\min }^{2}$ where the $\varepsilon_{\max } \sim \max E_{1}(p)-\min E_{0}(p)$ and $g_{\min }=\min g(p)$, the ground state of $H_{I}$ is evolved to the state which is with high probability the ground state of $H_{P}=H(p=1)$. Thus, we have prepared the system of the qubits in the ground state of Hamiltonian $H_{P}$ and they can be read-out. As a matter of fact the system is in the ground state of the Hamiltonian $H(p)$ during the whole adiabatic evolution, i.e. the system is immune against dephasing and relaxation. Here we should emphasize that the adiabatic evolution of the Hamiltonian is crucial in speeding up considerably the finding of the ground state of the Hamiltonian $H_{P}$. One could suggest that it is enough to wait a while and the system would relax itself into the ground state. However, a Hamiltonian which encodes a NP problem exhibits a 
lot of local minima and the physical system needs an exponentially long time (as a function of the number of qubits) to find its global minimum. As an example, one can consider Ising model of $N$ antiferromagnetically coupled magnetic moments. It is well known that such a system can be highly frustrated. The task of finding the minimum of the Ising Hamiltonian is equivalent to the optimization MAXCUT problem which belongs to a NPcomplete problem. ${ }^{24}$ Thus, it seems that NP problems cannot be solved in polynomial time on either digital or analog classical computers. Theoretically it was shown ${ }^{25}$ that an adiabatic quantum algorithm can find the global minimum of some functions in polynomial time whereas a classical simulated annealing algorithm requires exponential time. The crucial condition for adiabatic quantum evolution is the existence of an energy gap between the ground and upper levels. This is the key difference between classical and quantum systems, thereby enabling an enormous speed up of adiabatic quantum algorithms over classical ones. The size of the energy gap limits the speed of adiabatic quantum evolution as we will show experimentally in the next section.

Adiabatic evolution can be demonstrated on a single qubit. Following the original paper by Farhi et al. ${ }^{9}$ we start from the initial Hamiltonian at $t=0$

$$
H_{I}=\Delta \sigma_{x} .
$$

Then we adiabatically evolve from $H_{I}$ to the problem Hamiltonian $H_{P}$ in time $\tau$

$$
H_{P}=\varepsilon(\tau) \sigma_{z} .
$$

This scheme can be implemented for a superconducting flux qubit. Near the degeneracy point $f=0$, the qubit can be described by the Hamiltonian

$$
H(t)=\varepsilon(t) \sigma_{z}+\Delta \sigma_{x} .
$$

At a bias $\varepsilon=0$, the two lowest levels of the qubit anticross (Fig. 3), with a gap of $2 \Delta$. By increasing $\varepsilon$ slowly enough, the qubit will adiabatically transform from the superposition state $(|0\rangle+|1\rangle) / \sqrt{2}$ to $|1\rangle$, but remains in the ground state. For $|\varepsilon(\tau)| \gg \Delta, \Delta$ diminishes and the Hamiltonian takes the form

$$
H(\tau)=\varepsilon(\tau) \sigma_{z} .
$$

However, if the bias changes in time $\varepsilon(t)=\lambda t$, the qubit can 'jump' from the ground state $|g\rangle$ to the excited state $|e\rangle$ with probability $P_{L Z}=\exp \left(-\pi \Delta^{2} / \hbar \lambda\right)$. This process, known as a Landau-Zener transition, ${ }^{26}$ would violate adiabatic evolution and, therefore, should be avoided. This puts constraints on the characteristic time $\tau$ of the adiabatic evolution which can globally be estimated as: $\tau \gg \hbar E_{J} / \Delta^{2}$. Consequently, $\tau$ can be considerably shorter if we take into account that a Landau-Zener transition takes place only in the $\Delta$ vicinity of the anti-crossing point. Thus, $\varepsilon(t)$ can be changed quickly except in the region close to anti-crossing point.

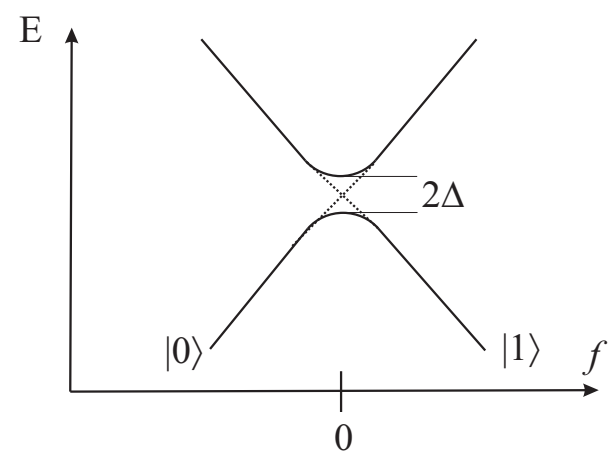

FIG. 3: Quantum energy levels of the qubit as a function of normalized internal magnetic flux $f=\Phi_{i} / \Phi_{0}-0.5$. For $f$ much less or greater than zero, the qubit is in the state $|0\rangle$ or $|1\rangle$,respectively. The dashed lines correspond to the classical potential minima.

For such a local adiabatic evolution the requirement for $\tau$ reads $\tau \gg \hbar / \Delta$. Note that only this condition leads to a quadratic speed-up of the adiabatic evolution version of Grover's algorithm. ${ }^{27} \mathrm{~A}$ measurement by a parametric transducer provides the unique possibility of controlling the speed of an adiabatic evolution. The smaller the energy gap is the larger is the signal from the transducer (see Eq.1,2). This signal can be used as feedback for $\varepsilon(t)$ sweeping so that the condition for adiabatic evolution can be satisfied locally for an unknown ground state of the system.

The tunnel splitting $2 \Delta$ is very sensitive to the Josephson and Coulomb energy of the junctions. It can be finely tuned by reducing the size of one junction in the superconducting loop, while leaving the two others unchanged. If the ratio between the area of the small and large junction is $\alpha(\alpha<1), \Delta$ can be roughly estimated ${ }^{3,23}$

$$
\begin{aligned}
\Delta & =\frac{E_{\mathrm{J}}}{\pi} \sqrt{\frac{2 \alpha-1}{\alpha g}} \\
& \times \exp \left[\sqrt{\frac{g(2 \alpha+1)}{\alpha}}\left(\arccos \frac{1}{2 \alpha}-\sqrt{4 \alpha^{2}-1}\right)\right],
\end{aligned}
$$

where $g=E_{J} / E_{C}$. By changing the parameters $\alpha$ and $g$, one obtains a crossover from the classical, through the Landau-Zener, to the adiabatic regime.

\section{B. Experiment}

In order to demonstrate the crossover from the classical to quantum regime we have prepared three qubits with different parameters $\alpha$ and $g$. The qubits were placed inside pancake niobium coil made by using electronbeam lithography on oxidized Si substrates. The typical linewidth and the distance between 20-30 coil windings are 1-2 $\mu \mathrm{m}$. The coils self-inductances are $L=$ $50-140 \mathrm{nH}$. For all experiments reported here we use an 
external capacitance $C=470 \mathrm{pF}$, therefore the tank resonance frequency is $19.6-32.8 \mathrm{MHz}$ with quality factors $\mathrm{Q}=700-1700$. The 3JJ qubit structure was fabricated out of $\mathrm{Al}$ in the middle of the coil by a conventional shadow evaporation technique. The critical current was determined, by measuring an rf-SQUID prepared on the same chip, as $I_{C}=250-400 \mathrm{nA}$. The qubit's loop area was 90 $\mu m^{2}$, with $L_{q}=40 \mathrm{pH}$. The typical coupling coefficient between the coil and qubit is $1-2 \times 10^{-2}$. In Fig. 2 the typical response of the inductive transducer is shown for three values of the parameters $\alpha$ and $g$, which correspond to three different regimes: classical, Landau-Zener and adiabatic. In classical regime the signal from parametric transducer is proportionate to the first derivative of the Josephson current with respect to internal magnetic flux. ${ }^{28}$ Close to degeneracy point there are two classical states corresponding to the currents flowing clockwise and counterclockwise (hysteretic behavior). For $g=60$ and $\alpha=0.9$ the qubit is in an intermediate regime where both tunneling between two classical states and LandauZener transitions are not negligible. There is still no visible dip in the phase characteristic but the losses caused by Landau-Zener transitions decrease the quality factor of the resonant circuit and, consequently, the amplitude of the $r f$ voltage. ${ }^{29}$ By keeping $g$ constant, but decreasing the size of the third junction from $\alpha=0.9$ to $\alpha=0.8$, the tunnel splitting $2 \Delta$ increases and Landau-Zener transitions are suppressed. As a result, a shift of the resonance frequency of the parametric transducer leads to huge dips in the $\theta$ vs $f$ curves (adiabatic regime). Nevertheless, if the voltage amplitude across the parametric transducer is increased high enough, the Landau-Zener transitions suppress the dip again. Under this condition, a discrepancy between experimental and theoretical curves, calculated within the adiabatic approach is observed (Fig. 4). Thus, we have observed the crossover from the classical, through the Landau-Zener, to the adiabatic regime of a superconducting flux qubit by decreasing the size of the Josephson junctions. Our experimental results show that the idea of adiabatic quantum computing can be demonstrated on a system of superconducting flux qubits. A reasonable and primarily feasible design is shown in the next section.

\section{IMPLEMENTATION OF THE MAXCUT PROBLEM FOR A SET OF INDUCTIVELY COUPLED SUPERCONDUCTING QUBITS}

The MAXCUT problem is a part of the NP-complete problems. Mathematically, in order to solve the MAXCUT problem, one should find the maximum of the payoff function $^{12}$

$$
P(|s\rangle)=\sum_{i} w_{i} s_{i}+\sum_{i, j} s_{i}\left(1-s_{j}\right) w_{i, j}
$$

where $w_{i j}, w_{i}$ are the parameters of the problem and $s_{i}=0,1$ are components of the vector $|s\rangle$. The problem

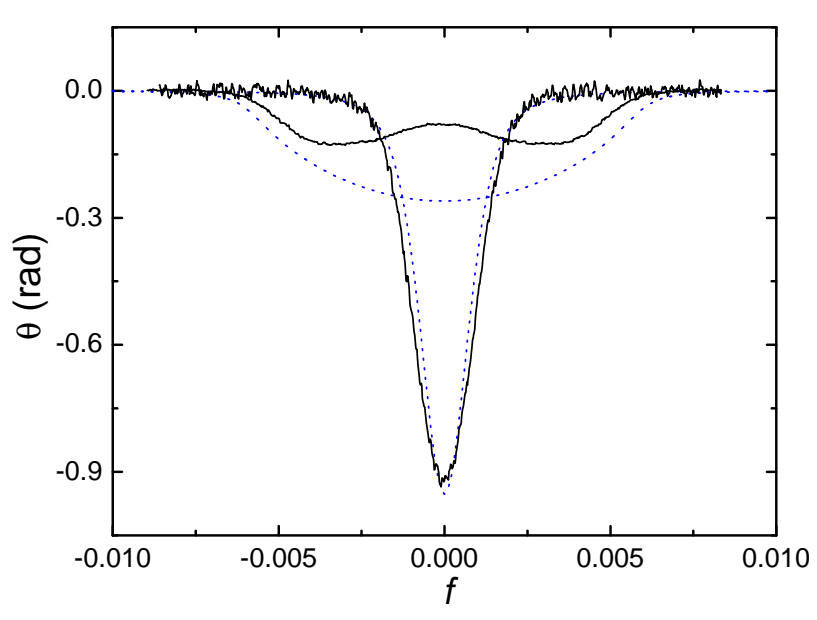

FIG. 4: The phase shift $\theta$ between the bias current $I_{r f}$ and the $r f$ voltage of the parametric transducer as a function of the normalized internal magnetic flux for small $V_{r f} \approx 0.5 \mu \mathrm{V}$ (lower curves) and large $V_{r f} \approx 5 \mu \mathrm{V}$ (upper curves) $r f$ voltages. The resonant frequency of the parametric transducer was $32 \mathrm{MHz}$. The discrepancy between experimental (solid line) and theoretical (dotted line) curves for the large amplitude $r f$ voltage is caused by Landau-Zener transitions.

can be encoded into a Hamiltonian $H$ of $N$ inductively coupled superconducting qubits

$$
H=\sum_{i=1}^{N} \varepsilon_{i}\left(f_{i}\right) \sigma_{z, i}+\sum_{i=1}^{N} \Delta_{i} \sigma_{x, i}+\sum_{i<j}^{N} J_{i, j} \sigma_{z, i} \sigma_{z, j}
$$

where $\sigma_{x}$ and $\sigma_{z}$ are Pauli matrices, $\varepsilon_{i}\left(f_{i}\right)$ is the energy bias of the $i$-th qubit, and $J_{i, j}$ is the coupling energy between the $i$-th and $j$-th qubit. The eigenvector $|s\rangle$, corresponding to the ground state of the Hamiltonian $H$, is the solution of the payoff function $P(|s\rangle)$ if (a) $\Delta_{i} \ll J_{i, j} \forall i, j$, and (b) $\varepsilon_{i}=-w_{i} / 2$, and $J_{i, j}=w_{i, j} / 2$.

For superconducting qubits, the initial Hamiltonian $H_{I}$ can be easily constructed by taking into account that $J_{i, j}=0$ and $\Delta_{i}=0$ if $f_{i}=-0.5$, i.e.

$$
H_{I}=\sum_{i=1}^{N} \varepsilon_{i}(-0.5) \sigma_{z, i}
$$

The ground state of $H_{I}$ is trivial, $|0\rangle$. By changing the bias of individual qubits adiabatically to $\varepsilon_{i}=-w_{i} / 2$, the $H_{I}$ is transformed to $H$. (The coefficients $w_{i, j}$ are set by design and they are determined by coupling energies between qubits.) $H$ encodes the payoff function $P(|s\rangle)$ completely if $\Delta_{i}=0$. Unfortunately, we cannot switch off the tunnel splitting $\Delta_{i}$ in superconducting qubits, but it is not absolutely necessary if $J_{i, j} \gg \Delta_{i}$. Nevertheless, we will show that by making use of a parametric transducer, ${ }^{18}$ one can obtain the answer even if $J_{i, j} \gtrsim \Delta_{i}$. Moreover, the qubit states can be readout while staying in the ground state of the system.

The most simple but still reasonable example of the adiabatic quantum optimization algorithm MAXCUT 


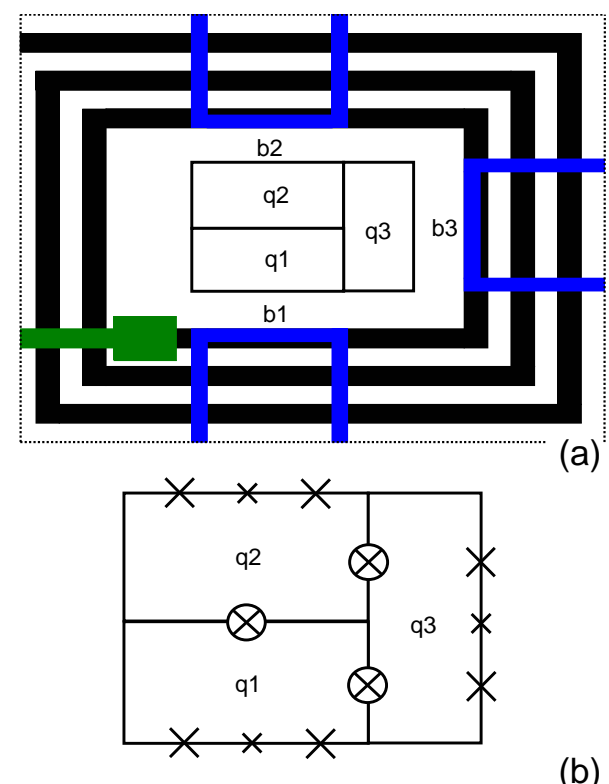

FIG. 5: Three-Qubit design for MAXCUT problem. (a) Three superconducting flux qubits are placed in superconducting coil. The qubits can be biased independently by $d c$ bias wires $b 1, b 2, b 3$. (b) The qubits are coupled through common Josephson junction marked by circles. The coupling energy $^{30} J_{i, j}=\left(M_{i, j}+\Phi_{0} / 2 \pi I_{c}\right) I_{q i} I_{q j}$, where $I_{c}$ is Josephson critical current of the common junction.

can be implemented by three coupled superconducting flux qubits $(N=3)$. The coupling between the qubits can be realized by means of a common Josephson junction ${ }^{30}$ shared between two qubits (see Fig. 5). This enable to increase the coupling energy over pure magnetic one. The coupling energy $\approx 0.3 \mathrm{~K}$ has been measured recently. ${ }^{31}$ Thus, for the present design we have chosen the interaction energies between the qubits to be $J_{1,2}=J_{2,3}=J_{1,3}=0.3 \mathrm{~K}$, the persistent currents are $I_{p 1}=I_{p 3}=350 \mathrm{nA}, I_{p 2}=420 \mathrm{nA}$ and tunneling matrix elements are $\Delta_{1}=\Delta_{2}=\Delta_{3}=96 \mathrm{mK}$. By choosing appropriate values for $\varepsilon_{i}$ it is possible to realize the situation that the system exhibits both a local and a global minimum. We have chosen the following parameters $\varepsilon_{1}(0.006)=0.315 \mathrm{~K}, \varepsilon_{2}(0.004)=0.252 \mathrm{~K}$, and $\varepsilon_{3}(0.01)=0.525 \mathrm{~K}$. The energy of the ground state for various vectors $|s\rangle$ is shown in Table I. In the state $|101\rangle$ the system is in the global minimum. Note that for $|110\rangle$ the system exhibits a local minimum, that is, there is no way to decrease the energy of the system by flipping the persistent current in one qubit only. Thus, the system can stay in the state $|110\rangle$ for an exponentially long time at low temperatures. In our design the lowest 'energy' barrier which the system sees from the local minimum is higher than $0.5 \mathrm{~K}$. This could lead to a wrong answer, unless the Hamiltonian transform is carried out adiabatically.

The qubits' state can be readout by an inductive transducer as was described above. The internal magnetic

\begin{tabular}{c||c|c|c|c|c|c|c|c}
$|s\rangle$ & 000 & 010 & 011 & 001 & 101 & 111 & 110 & 100 \\
\hline$E(\mathrm{~K})$ & 1.992 & 0.288 & -0.342 & 0.162 & -0.889 & -0.192 & -0.762 & -0.258
\end{tabular}

TABLE I: Energy of the system for various vectors. $J_{1,2}=$ $J_{2,3}=J_{1,3}=0.3 \mathrm{~K} . \varepsilon_{1}=0.315 \mathrm{~K}, \varepsilon_{2}=0.252 \mathrm{~K}$, and $\varepsilon_{3}=$ $0.525 \mathrm{~K}$.

flux of the individual qubits can be changed by a current through the wires placed nearby each of them. In such a configuration, all three qubits can be readout by making use of one transducer only. Nevertheless, the idea should be checked since qubits interact and $\Delta$ is nonzero. The three qubit Hamiltonian can be solved numerically. In the following section we simulate the readout of a parametric transducer inductively coupled to three superconducting flux qubits.

\section{A. Numerical simulation}

The Hamiltonian (17) was solved numerically and the energy levels of the Hamiltonian (17) as a function of $f_{i}$ are shown in Fig. 6. We have used the same parameters as those used in our design. We have also calculated the response of the parametric transducer using the formula 32,33

$$
\tan \theta=-2 Q \sum_{\nu} \frac{R_{0 \nu}}{E_{\nu}-E_{0}}
$$

where $E_{\nu}-E_{0}$ is the distance between ground and upper energy levels, and

$$
\begin{aligned}
R_{0 \nu}= & \left(\sum_{i=1}^{N} k_{i} \sqrt{L_{q i}} I_{q i}\left\langle 0\left|\sigma_{z}^{(i)}\right| \nu\right\rangle\right) \\
& \times\left(\sum_{j=1}^{N} k_{j} \sqrt{L_{q j}} I_{q j}\left\langle\nu\left|\sigma_{z}^{(j)}\right| 0\right\rangle\right)
\end{aligned}
$$

are the real matrix elements. Here $k_{i}$ is the coupling coefficient between the $i$-th qubit and resonator, and $L_{q i}$ is the qubit's inductance. For $Q=1000, L=81 \mathrm{nH}$, $L_{q i}=40 \mathrm{pH}$, and $k_{i}=0.036$ the results are shown in Fig. 7. From these figures it is apparent that the qubits' states can be determined by a parametric transducer. We have also tried to find the threshold for $\Delta_{i}$ below which the state of the qubit cannot be distinguished. As a criterion the existence of the distinguishable dips on the experimental curves can be chosen. From Fig. 8 one can see that the positions of the dips do not change as $\Delta$ increases and they can be distinguished for relatively large values of $\Delta$. Thus, the parametric transducer readout delivers the right solution of the problem.

\section{CONCLUSIONS}

Experimentally, we have demonstrated the principle of adiabatic quantum evolution in a single qubit. Theo- 

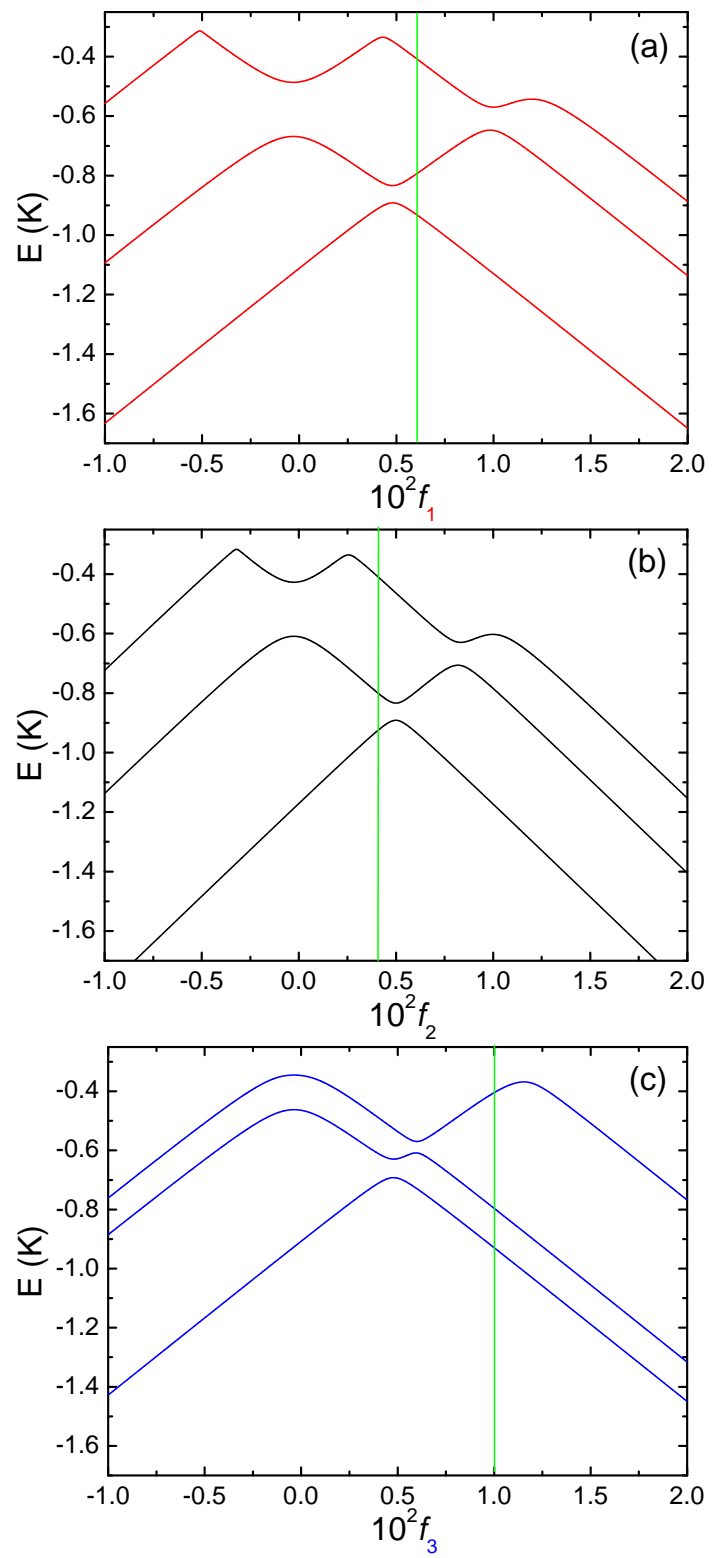

FIG. 6: First three energy levels of the three qubit system during readout. Readout of the qubit starts at point $f_{1}^{p}=$ $0.006, f_{2}^{p}=0.004$, and $f_{3}^{p}=0.01$ then its bias is changed adiabaticaly and separately through qubit 1 (a), 2 (b), and 3 (c) while keeping it fixed in the others. At the points with a large curvature of the ground level the parametric transducer gives a considerable response (see Fig. 7). If this point is on the left (right) side of the point corresponding to the problem Hamiltonian (marked by green vertical line) the qubit is (or better to say would be if $\Delta=0)$ in the state $|0\rangle(|1\rangle)$.

retically, we have shown that three inductively coupled superconducting flux qubits placed in a superconducting coil can be used to demonstrate the adiabatic quantum algorithm MAXCUT which belongs to the set of NPcomplete problems. A three qubit design has been proposed and simulated numerically.

Note added in proof: Recently, Lupascu et al. [A.

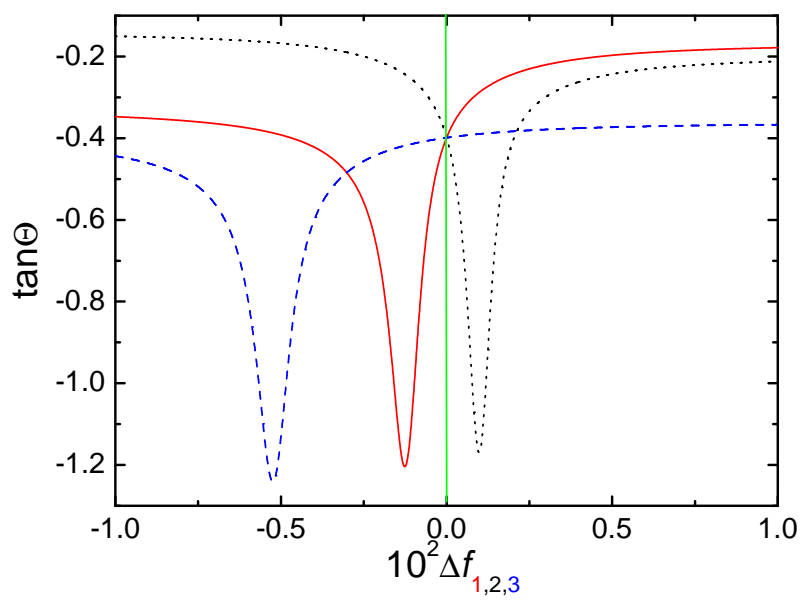

FIG. 7: The phase shift between the voltage and bias current of the parametric transducer with respect to $\Delta f=f-f^{p}$. Readout of the qubit starts at point $f_{1}^{p}=0.006, f_{2}^{p}=0.004$, and $f_{3}^{p}=0.01$ then its internal magnetic flux is swept adiabaticaly around this point. The red (solid), black (dotted) and blue (dashed) lines correspond to bias flux change in qubit 1,2 and 3 , respectively. From the position of the dips we find that the state $|101\rangle$ corresponds to the global minimum (compare with Table I).

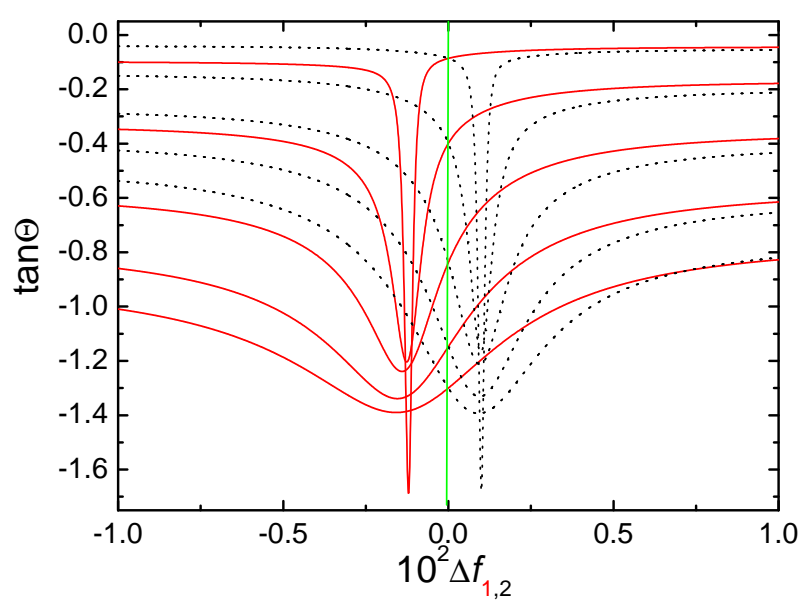

FIG. 8: The phase shift between voltage and bias current of the inductive transducer with respect to $\Delta f=f-f^{p}$ for various values of $\Delta_{i}\left(\Delta_{i}\right.$ is taken to be the same for all qubits). Readout of the qubit starts at point $f_{1}^{p}=0.006, f_{2}^{p}=$ 0.004 , and $f_{3}^{p}=0.01$ then the internal magnetic flux is swept adiabaticaly around this point. The red solid line and black dotted line correspond to the qubits 1 and 2, respectively. From the upper to lower curve (at $\Delta f=0.01$ ) $\Delta_{i}$ takes the values $0.048,0.096,0.144,0.192$, and $0.240 \mathrm{~K}$.

Lupascu, C. J. M. Verwijs, R. N. Schouten, C. J. P. M. Harmans, and J. E. Mooij, Phys. Rev. Lett. 93, 177006 (2004)] proposed a similar readout method which enables to measure the observable $\sigma_{z}$ in a nondestructive way. 


\section{ACKNOWLEDGEMENT}

The authors thank D-Wave Systems Inc. for financial support and M.H.S. Amin, D.V. Averin, A. Blais, Alec Maassen van den Brink, Ya.S. Greenberg, H.E. Hoenig,
H.-G. Meyer, Yu.A. Pashkin, A. Shnirman, F.K. Wilhelm, and A.M. Zagoskin for fruitful discussions. M.G. wants to acknowledge partial support by Grant Nos. VEGA 1/2011/05 and APVT-51-016604.
* Electronic address: grajcar@fmph.uniba.sk

1 P. Shor., Algorithms for Quantum Computation: Discrete Logarithms and Factoring. In Proc. 35th Annual Symposium on Foundations of Computer Science., (IEEE Press, Los Alamitos, 1994).

2 J.E. Mooij, T.P. Orlando, L. Levitov, L. Tian, C.H. van der Wal, and S. Lloyd, Science 285, 1036 (1999).

3 T.P. Orlando, J.E. Mooij, L. Tian, C.H. van der Wal, L. Levitov, S. Lloyd, and J.J. Mazo, Phys. Rev. B 60, 15398 (1999)

${ }^{4}$ C.H. van der Wal, A.C.J. ter Haar, F.K. Wilhelm, R.N. Schouten, C.J.P.M. Harmans, T.P. Orlando, S. Lloyd, and J.E. Mooij, Science 290, 773 (2000).

5 Y. Nakamura, Yu.A. Pashkin, and J.S. Tsai, Nature 398, 786 (1999); J.R. Friedman, V. Patel, W. Chen, S.K. Tolpygo, and J.E. Lukens, Nature 406, 43 (2000); J.M. Martinis, S. Nam, J. Aumentado, and C. Urbina, Phys. Rev. Lett. 89, 117901 (2002); D. Vion, A. Aassime, A. Cottet, P. Joyez, H. Pothier, C. Urbina, D. Esteve, and M.H. Devoret, Science 296, 886 (2002);

6 I. Chiorescu, Y. Nakamura, C.J.P.M. Harmans, J. E. Mooij, Science 299, 1869 (2003).

7 E. Il'ichev, N. Oukhanski, A. Izmalkov, Th. Wagner, M. Grajcar, H.-G. Meyer, A.Yu. Smirnov, A. Maassen van den Brink, M.H.S. Amin, and A.M. Zagoskin, Phys. Rev. Lett. 91, 097906 (2003).

8 M. Oskin, F.T. Chong, and I.L. Chuang, IEEE Computer 35, 79 (2002).

9 E. Farhi, J. Goldstone, S. Gutmann, M. Sipser, quantph/0001106.

10 W.M. Kaminsky, S. Lloyd, and T.P. Orlando, quant$\mathrm{ph} / 0403090$.

11 D. Aharonov, Wim van Dam, Julia Kempe, Z. Landau, S. Lloyd, O. Regev, quant-phys/0405098.

12 M. Steffen, Wim van Dam, T. Hogg, G. Breyta, and I. Chuang, Phys. Rev. Lett. 90, 067903 (2003).

13 V.B. Braginsky and F.Ya. Khalili, Quantum Measurement, edited by Kip S. Thorne (Cambridge University Press, Cambridge, 1992).

14 N. Oukhanski, M. Grajcar, E. Il'ichev, and H.-G. Meyer, Rev. Sci. Instr. 74, 1145 (2003).

15 A.H. Silver and J.E. Zimmerman, Phys. Rev. 157, 317 (1967).

16 R.A. Buhrman, SQUID'76, edited by H.D Hahlbohm and H. Lübbig, (Walter de Gruyter, Berlin, 1977), p. 395.
17 A.Yu. Smirnov, Phys. Rev. B 68, 134514 (2003).

18 M. Grajcar, A. Izmalkov, E. Il'ichev, Th. Wagner, N. Oukhanski, U. Hübner, T. May, I. Zhilyaev, H.E. Hoenig, Ya.S. Greenberg, V.I. Shnyrkov, D. Born, W. Krech, H.-G. Meyer, Alec Maassen van den Brink, and M.H.S. Amin, Phys. Rev. B 69, 060501(R) (2004).

19 The analogy between spin and the Stern-Gerlach apparatus on the one hand and persistent current and SQUID on the other is not complete since rotating the SQUID by $90^{\circ}$ does not allow the measurement of $\sigma_{x}$.

20 H. Haken, Light, (Elsevier Science, Amsterdam, 1981) p. 208.

21 A. Blais, R. Huang, A. Wallraff, S. M. Girvin, and R. J. Schoelkopf, Phys. Rev. A 69, 062320 (2004).

22 D.V. Averin, Phys. Rev. Lett. 88, 207901 (2002).

23 Ya.S. Greenberg, A. Izmalkov, M. Grajcar, E. Il'ichev, W. Krech, H.-G. Meyer, M.H.S. Amin, and Alec Maassen van den Brink, Phys. Rev. B 66, 214525 (2002).

24 M.R. Garey, D.S. Johnson, and L. Stockmeyer, Theor. Comput. Sci. 1, 237 (1976).

25 E. Farhi, J. Goldstone, S. Gutmann, quant-ph/0201031.

26 L. D. Landau, Z. Phys. Sowjetunion 2, 46 (1932); C. Zener, Proc. R. Soc. London A 137, 696 (1932).

27 J. Roland and N.J. Cerf, Phys. Rev. A 65, 042308 (2002).

28 E. Ilichev, Th. Wagner, L. Fritzsch, J. Kunert, V. Schultze, T. May, H. E. Hoenig, H.-G. Meyer, M. Grajcar, D. Born, W. Krech, M. V. Fistul, and A. M. Zagoskin, Appl. Phys. Lett. 80, 4184 (2002).

29 A. Izmalkov, M. Grajcar, E. Il'ichev, N. Oukhanski, Th. Wagner, H.-G. Meyer, W. Krech, M.H.S. Amin, Alec Maassen van den Brink, and A.M. Zagoskin, Europhys. Lett. 65, 844 (2004).

30 L. S. Levitov, T. P. Orlando, J. B. Majer, and J. E. Mooij, cond-mat/0108266; Alec Maassen van den Brink, private communication.

31 M. Grajcar, A. Izmalkov, S.H.W. van der Ploeg, S. Linzen, E. Il'ichev, Th. Wagner, U. Hubner, H.-G. Meyer, Alec Maassen van den Brink, S. Uchaikin, A.M. Zagoskin, condmat/0501085 (unpublished).

32 A. Izmalkov, M. Grajcar, E. Ilichev, Th.Wagner, H.G. Meyer, A.Yu. Smirnov, M.H.S. Amin, Alec Maassen van den Brink, and A.M. Zagoskin, Phys. Rev. Lett 93, 037003 (2004).

33 A.Yu. Smirnov, cond-mat/0312635. 